\title{
10 Cloud-Dienste
}

\subsection{Cloud: Vor- und Nachteile}

Im Grunde handelt dieses ganze Buch vom Arbeiten „in der Cloud“, da alle Web 2.0-Anwendungen auf Servern im Netz liegen und man somit im Netz arbeitet. Wenn man nicht als Profi(-Programmierer), sondern als Laie im Netz unterwegs ist, dann versteht man unter „Cloud“ meist die Möglichkeit, im Netz Daten abzulegen und zwischen verschiedenen Geräten zu synchronisieren. In diesem Sinne möchten wir Ihnen in diesem Kapitel Dienste und Möglichkeiten vorstellen, die wir bisher noch nicht behandelt haben, insbesondere die Dateiablage und das Teilen von Dateien im Netz.

\subsubsection{Hosting}

Bei den meisten Cloud-Diensten liegt der Firmensitz und somit in der Regel auch der Ort der Datenspeicherung in den USA. Für die auf den Servern von Cloud-Diensten gespeicherten Daten gelten jeweils die Datenschutzrichtlinien des Landes, in dem der Server steht. Je nachdem, wie sensibel Ihre Daten sind, sollten Sie also prüfen, in welchem Land und somit unter welchen Datenschutzrichtlinien Ihre Daten gespeichert werden.

\subsection{Dienste}

\subsubsection{Dropbox}

Dropbox [www.dropbox.com] bietet Ihnen die Möglichkeit, im Netz in einem geschützten Raum Verzeichnisse an- und Dateien abzulegen. Bis zu 2 GB sind kostenlos, mehr Speicherplatz kann man sich in 0,5 GB-Schritten durch Werbung ,verdienen“, alles, was darüber hinausgeht, ist kostenpflichtig. Es gibt viele vergleichbare Dienste, Dropbox hat sich seit zwei Jahren seinen Ruf dadurch verdient, dass es besonders funktional einzusetzen ist:

- man kann durch die Installation eines kleinen Zusatzprogrammes die DropboxOrdner in die Verzeichnishierarchie des Computers „einhängen“ und dann Dateien im Explorer, in Dateicontrollern oder beim Abspeichern so behandeln wie sonst auch. Den Unterschied, dass die Datei im Netz gespeichert/aus dem Netz geholt wird, merkt man nicht.

- durch verschiedene Apps ist es auch für iPhone, iPad, Android-Smartphones und Blackberry-Geräte nutzbar.

- man kann einzelne Ordner für bestimmte Nutzer freigeben, d. h. für Arbeitsgruppen oder Abteilungen ist so eine Ablage für Dateien möglich. Wer also kein Intranet seitens der Institution zur Verfügung hat oder für Kooperationen eine gemeinsame Ablage benötigt, der kann hier leicht Entsprechendes einrichten. So ist auch gewährleistet, dass alle immer auf die aktuelle Datei zugreifen.

Dienste wie Dropbox machen den USB-Stick so gut wie überflüssig, halten den Datenbestand synchron und stellen so eine erhebliche Arbeitserleichterung dar. Außerdem bieten sie ein Back-up Ihrer Dateien, für den Fall, dass Sie die Originaldateien z.B. durch einen technischen Defekt verlieren.

\section{Lernziele}

In diesem Kapitel lernen Sie, wie man Dateien auf einem Server im Netz abzulegen, diese Ablage zu strukturieren, einzelne Ordner mit anderen zu teilen und Backuproutinen mit Hilfe von Cloud-Diensten einzurichten.

Inhalt dieses Kapitels:

- Vor- und Nachteile von Clouddiensten

- Dropbox als die zur Zeit beliebteste Anwendung in diesem Bereich

- Sugarsync als eine zweite Option

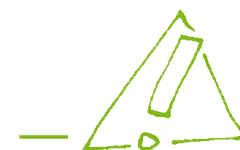

Wichtig zu wissen: Zwei Ihrer Dropbox-Ordner sind automatisch öffentlich zugänglich, „Photos“ und „Public“. Sie sind vom Ordnersymbol her auch anders gekennzeichnet. Wenn Sie Fotos ablegen und dies nicht öffentlich machen wollen, sollten Sie unbedingt einen Extraordner anlegen. 


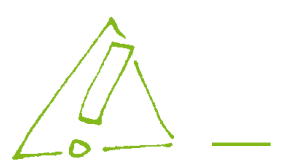

Der Datenschutz ist zwar gewährleistet, jedoch nicht auf höchster Stufe. Es gab schon einmal Vorkommnisse, dass Zugänge zu Accounts temporär möglich waren, und die US-Behörden haben auch Zugang zu Ihren Daten/Dateien! Man sollte also nichts Vertrauliches dort lagern.

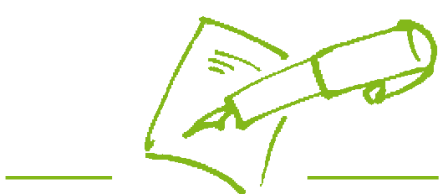

Eine kleine Übung

Wenn Sie oben in Kapitel 2 die Möglichkeiten gelesen haben, den Browser mit Hilfe von Add-ons zu erweitern, müsste Ihnen SimilarSites aufgefallen sein. Installieren Sie es, falls Sie das noch nicht getan haben, laden dann im Browser Dropbox [www.dropbox.com] und drücken auf das Icon von SimilarSites. Nun sehen Sie, welche anderen Dienste derzeit eine ähnliche Dienstleistung anbieten wie Dropbox. Sicher ist auch Sugarsync darunter, das wir jetzt in der Folge besprechen.
So ist mir z. B. eine Woche vor einem Großevent, für das ich die Projektverantwortung und -koordination innehatte, meine Festplatte komplett kaputt gegangen. Es war nichts mehr zu retten. Dies ist normalerweise der Moment, wo einem bewusst wird, dass man schon länger vorhatte, ein Back-up zu machen und man so richtig ins Schwitzen gerät. Da ich aber alle Projektdateien in der Cloud gespeichert hatte, konnte ich schnell eine neue Festplatte einbauen lassen, habe den Rechner anschließend mit meiner Cloud verbunden und synchronisiert und alles war wieder da. Über das Web hatte ich auch während der Reparatur meines Rechners jederzeit Zugriff auf meine Dateien. Es ist für mich also nicht zu einer „Zwangsarbeitspause“ gekommen, weil mir die Unterlagen jederzeit zur Verfügung standen.

\section{Verwaltung}

Um Ihre Dateien auf Ihrem Rechner zu verwalten, installieren Sie den Desktop-Client von Dropbox. Der Desktop-Client ist für Windows, Mac und Linux verfügbar. Der Dropbox-Client verhält sich wie ein Ordner in Ihrer Dateiverwaltung. Alle Bibliotheken und Ordner, die Sie hier hineinlegen, werden automatisch mit dem Dropbox-Server und allen weiteren angeschlossenen Endgeräten synchronisiert. Über das Kontextmenü, das sich beim Rechtsklick auf die Ordner/Dateien im Dropbox-Ordner öffnet, haben Sie die Option, Dateien freizugeben oder einen sicheren Link zum Teilen zu generieren.

Die Hauptverwaltung Ihres Accounts, Freigabe von Ordnern sowie die Verwaltung der angeschlossenen Geräte und eine Übersicht der Apps, die auf Dropbox zugreifen, befindet sich auf der Dropbox Webseite.

Hier finden Sie auch die Angaben zum verbrauchten Speicherplatz und die Option, weiteren Speicherplatz hinzuzukaufen.

\section{App}

Dropbox bietet Apps für IOS, Android und Blackberry an. Zusätzlich gibt es eine ganze Reihe von Drittanbietern, die den Zugriff auf Dropbox zum Herunterladen und/ oder Exportieren aus Ihren Anwendungen anbieten.

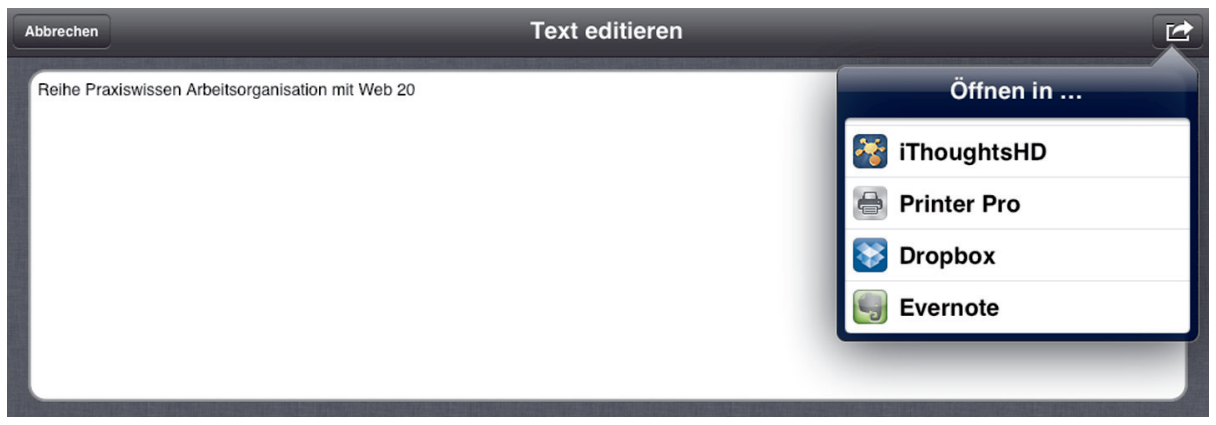

Dropbox unterscheidet seine Synchronisationsroutine zwischen Rechnern und Apps. Bei angeschlossenen Rechnern werden alle Daten komplett synchronisiert. Die App hingegen lädt nur die Verzeichnisstruktur, nicht aber die gesamten Dateien. Aus der Verzeichnisstruktur können Dateien ausgewählt werden, die dann ad hoc auf das mobile Endgerät herunter geladen werden. Dies hält das Datenvolumen klein. Sie können einzelne Dateien als Favoriten markieren. Diese Dateien werden dann immer als ganze Dateien synchronisiert und stehen Ihnen so auch im Offline-Modus jederzeit zur Verfügung. 


\subsubsection{Sugarsync}

Sugarsync [sugarsync.com] ist ebenfalls ein sehr beliebter Cloud-Dienst. Während Dropbox 2 GB frei zur Verfügung stellt, stellt Sugarsync 5 GB frei zur Verfügung. Der größte Unterschied zwischen Sugarsync und Dropbox liegt jedoch in der Art, wie die Dienste die Dateien/Ordner für die Synchronisation verwalten.

\section{Verwaltung}

Während man bei Dropbox einen Ordner hat, dessen Inhalt komplett mit allen Endgeräten snychronisiert wird, hat Sugarsync einen eigenen Desktop-Client für die Verwaltung der Dateien, der es möglich macht, pro Endgerät festzulegen, welche Ordner bzw. Dateien synchronisiert werden sollen.

Die Ordner bleiben an ihrem Platz in der Dateiverwaltung Ihres Rechners und werden im Sugarsync Desktop-Client, dem Sugarsync-Dateimanager, nur für die Synchronisation markiert. Dabei kann man entscheiden, welche Ordner nur mit der Cloud synchronisiert werden sollen und welche Ordner und Dateien mit anderen Endgeräten synchronisiert werden sollen. Zentraler Speicherort, mit dem sich alle Endgeräte synchronisieren, ist auch hier der Sugarsync-Server.

Auch bei Sugarsync können Sie Ordner und Dateien mit anderen teilen. Der Sugarsync-Dateimanager ist für Windows und Mac verfügbar, nicht aber für Linux.

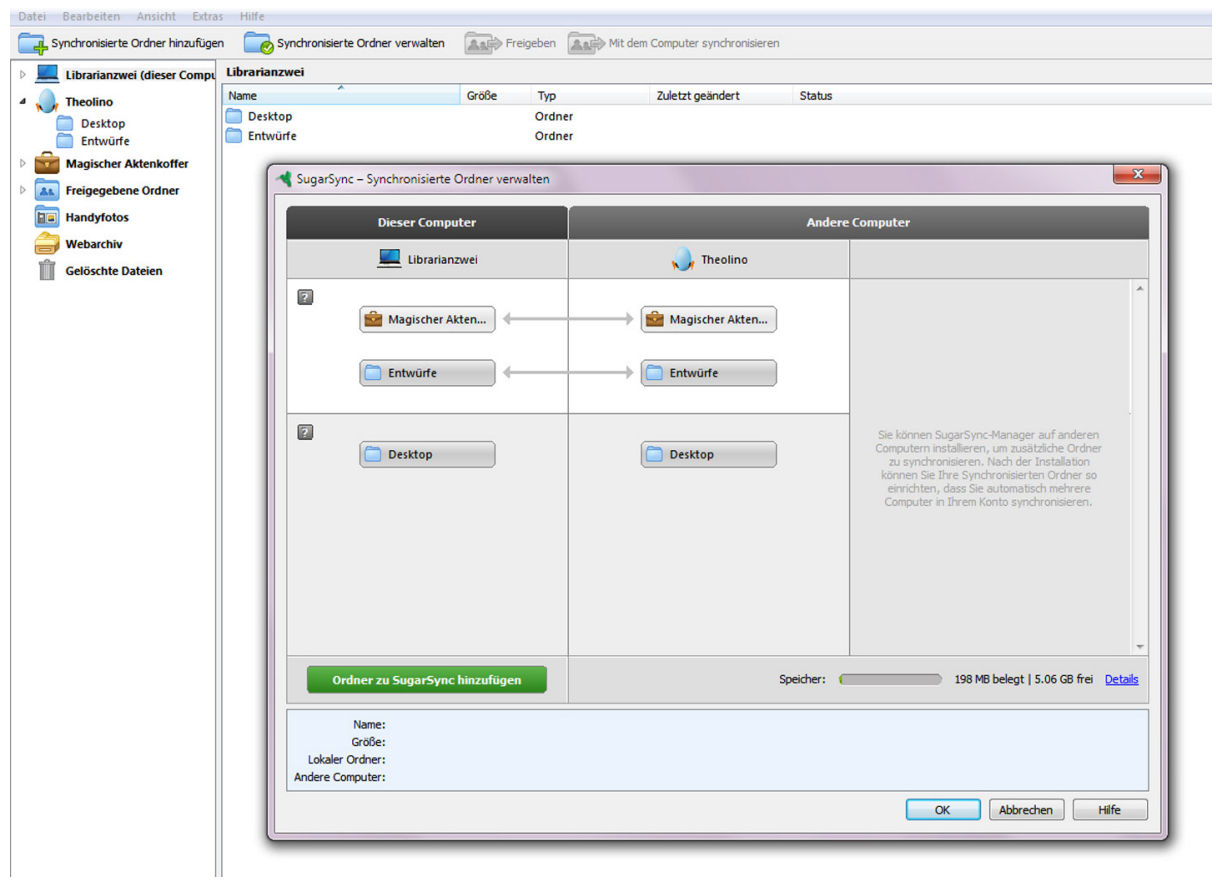




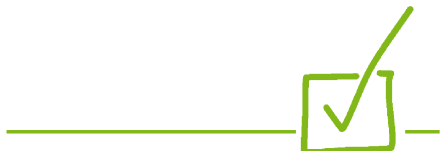

Tipp

Neben den hier vorgestellten CloudDiensten gibt es natürlich noch eine Reihe weiterer Dienste. Darunter auch anbietergebundene Dienste, wie z. B. iCloud für alle AppleGeräte und die Telekom-Cloud für Telekomkunden.
In der Dateiverwaltung von Sugarsync gibt es einen Sonderordner, der sich „magischer Aktenkoffer" nennt. Dateien, die hier abgelegt werden, werden immer auf alle Endgeräte synchronisiert. Für diesen Ordner kann man sich dann z. B. eine Verknüpfung auf dem Desktop machen, um Dateien, die gerade bearbeitet werden, schnell dort ablegen zu können. Dann hat man sie unterwegs auf jedem Gerät zur Hand.

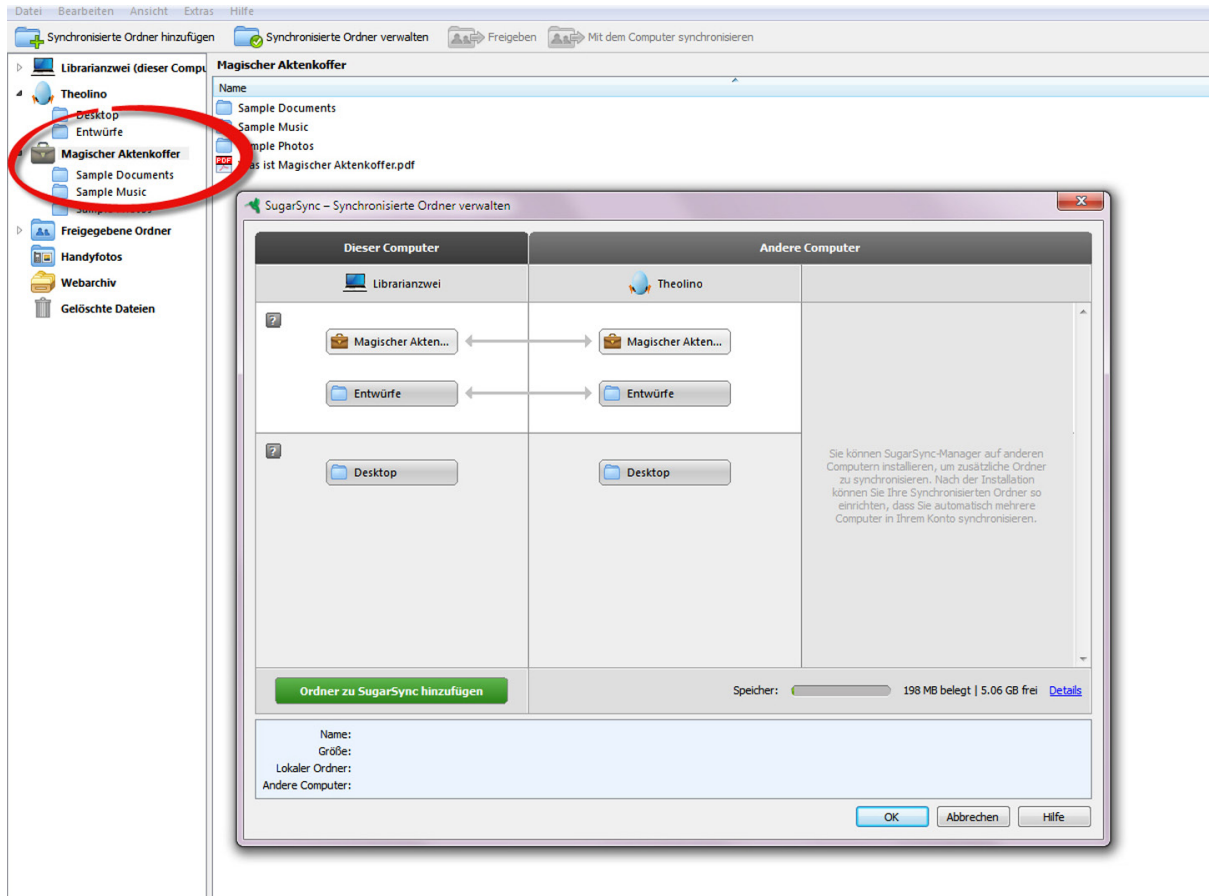

Insgesamt muss man sich zunächst in die Verwaltung der verschiedenen Geräte über den Sugarsync-Dateimanager bzw. die Apps etwas einarbeiten. Dann bietet Sugarsync für den anspruchsvollen Anwender in puncto Dateiverwaltung mehr Features als Dropbox.

Wie schon bei Dropbox, ist die zentrale Verwaltung Ihres Nutzer-Accounts auch hier auf der Sugarsync Webseite zu finden.

\section{App}

Sugarsync bietet seine App für eine ganze Anzahl Endgeräte/Betriebssysteme an. Dazu gehören:

- Blackberry

- Android

- IOS

- Nokia (Symbian)

- Windows Mobile

Von der App kann man entweder auf Dateien in der Cloud zugreifen und diese herunterladen oder auf die standardmäßig mit dem Endgerät synchronisierten Dateien zurückgreifen. Diese Dateien stehen auch offline zur Verfügung.

Sie können über die Sugarsync Webseite auch Dateien an Ihr mobiles Endgerät schicken, damit diese Ihnen dort direkt zur Verfügung stehen. 
Standardisierte Synchronisationsroutinen einrichten mit ifttt

Der Webdienst ifttt (if this, than that [iftt.com]) bietet Ihnen die Möglichkeit, feste Abläufe für den Transfer Ihrer Daten von einem Cloud-Dienst zum anderen zu definieren. Dabei können Sie entweder selbst Routinen (Tasks) definieren oder sich aus der umfangreichen Rezeptbibliothek bedienen.

So können Sie z. B. definieren:

- Dass immer wenn Sie einen Artikel im Google-Reader mit einem Stern markieren, dieser automatisch an ein definiertes Notizbuch in Evernote gesandt wird.

- Oder dass Sie jedem, der Sie auf Twitter erwähnt, automatisch danken.

- Oder dass automatisch alle Fotos, die Sie zu Facebook hochladen, auch in Ihrer Dropbox gespeichert werden.

Uvm.

Die Verwendung dieses Dienstes kann Ihnen viel manuelle Einzelarbeit abnehmen und ist einfach zu bedienen. 